\title{
Avaliação Colorimétrica de Aglomerados Produzidos com Partículas de Madeira de Sequoia sempervirens e Pinus taeda Submetidos ao Intemperismo Natural
}

\author{
Vinicius Gomes de Castro ${ }^{1}$, Rafael Leite Braz ${ }^{1}$, Karine Bellon ${ }^{1}$, \\ Felipe Sanches ${ }^{1}$, Setsuo Iwakiri ${ }^{1}$, Silvana Nisgoski ${ }^{1}$ \\ ${ }^{1}$ Departamento de Engenharia e Tecnologia Florestal, Universidade Federal do Paraná - UFPR, Curitiba/PR, Brasil
}

\begin{abstract}
RESUMO
O objetivo do presente trabalho foi estabelecer o efeito do uso de misturas de diferentes proporções de partículas de Sequoia sempervirens e Pinus taeda nas propriedades colorimétricas de aglomerados, além de avaliar a alteração natural da cor após exposição ao intemperismo natural por seis meses. As análises colorimétricas foram realizadas através do sistema CIE-L*a* $b^{\star}$ 1976. Os resultados indicaram uma relação direta das proporções de partículas de Sequoia sempervirens com os valores médios do pigmento vermelho $\left(\mathrm{a}^{*}\right)$ e uma relação indireta com os valores do pigmento amarelo $\left(b^{*}\right)$. Desta maneira, a cor do produto final pode ser prevista de acordo com o controle de proporções de partículas de diferentes espécies. Contudo, a exposição do material à luz natural por quatro meses é o suficiente para afetar negativamente os pigmentos $\mathrm{a}^{\star} \mathrm{e} \mathrm{b}^{\star}$, e tende a eliminar a diferença de cores entre os painéis.
\end{abstract}

Palavras-chave: colorimetria, aglomerados crus, CIE-L*a* $\mathrm{b}^{\star}$.

\section{Outdoor Weathering Evaluation of Particleboards Manufactured from Sequoia sempervirens and Pinus taeda Wood}

\begin{abstract}
The aim of this work was to establish the effect of using different amounts of Sequoia sempervirens and Pinus taeda wood particles on the colorimetric properties of particleboard and to evaluate the color change after outdoor weathering for six months. The colorimetric properties were measure according to the CIE-L*a* $\mathrm{b}^{*} 1976$ system. The results indicated a direct relation between the proportion of Sequoia sempervirens wood particles used in the blend and the red pigment values $\left(\mathrm{a}^{\star}\right)$ and an indirect relation with the yellow pigment $\left(\mathrm{b}^{*}\right)$. Thus the final color of a particleboard can be predicted by controlling the amount of particles from the different species used in particleboard production. However, natural weathering for 4 months is sufficient to decrease $\mathrm{a}^{\star}$ and $\mathrm{b}^{\star}$ values and eliminate the color difference between the particleboards.
\end{abstract}

Keywords: colorimetric properties, particleboard, CIE- $L^{\star} a^{\star} b^{\star}$. 


\section{INTRODUÇÃO}

A madeira é usada como elemento de decoração de um ambiente interno devido não somente às suas propriedades físicas, como resistência mecânica, durabilidade, custo e facilidade de usinagem, mas também pelo seu aspecto estético e pela capacidade de transmitir a sensação de conforto. Tsunetsugu et al. (2007) pesquisaram o efeito psicológico e fisiológico em jovens quando visualmente estimulados por quartos decorados com diferentes quantidades de madeira. Ambientes com $45 \%$ de elementos decorativos com madeira (piso e paredes cobertas por lâminas de madeira até a altura do peito) foram apontados pelos pesquisados como os mais "confortáveis" e "calmantes", sentimento confirmado pela redução da pressão sanguínea. Zaleski (2006) fez um levantamento na cidade de Curitiba com moradores de classe média e relatou que $43 \%$ dos entrevistados escolheram, entre fotografias de diferentes estilos de decoração para dormitórios, aquele que apresentava a parede revestida por painéis de madeira.

Os painéis de madeira podem ser definidos como produtos compostos de elementos, como lâminas, sarrafos, partículas e fibras, obtidos a partir da redução da madeira sólida e reconstituídos através de ligação adesiva (Iwakiri et al., 2005). Entre os principais painéis, encontram-se os aglomerados ou MDP (medium density particle boards), que podem ser comercializados crus - ou in natura - ou revestidos. Atualmente, há três tipos de revestimento, que empregam padrões madereiros ou em cores: o revestimento laminado de baixa pressão (BP), que usa uma folha de papel especial impregnada com resina melamínica fundida ao painel com calor e pressão; o finish foil (FF), que consiste na colagem de uma película de papel à chapa, e o revestimento de lâmina de madeira natural (LM) (Mattos et al., 2008). Contudo, os resíduos produzidos nas indústrias de revestimento não são passíveis de reciclagem. O papel FF possui verniz na sua camada exterior, impedindo a repolpagem, e o papel BP possui baixa concentração de celulose. Mensalmente, produzem-se cerca de $8,6 \mathrm{~kg}$ de resíduo para cada $1000 \mathrm{~m}^{2}$ de revestimento produzido (Solyon, 2009).

Considerando-se a preocupação com a responsabilidade ambiental e com a sustentabilidade, os designers de produtos devem estar atentos a materiais alternativos, processos industriais de menor impacto e degradação ambiental, escassez de recursos naturais e minimização de resíduos de fase pós-uso. Além disso, nos móveis fabricados a partir de uma consciência ecológica, geralmente respeita-se a coloração do próprio material e utilizam-se vernizes à base de água no revestimento (Rosa \& Gonçalves, 2010). Nascimento (2003) apresenta o uso de aglomerados feitos a partir de espécies da Região Nordeste do Brasil em sua forma crua, na criação de mesas e objetos de decoração, confirmando o uso do design para elevar o valor agregado do material.

Contudo, para que os diferentes tipos de aglomerado in natura possam ser trabalhados pelos designers, a análise da cor se torna uma exigência. Esta é um dos componentes da estética que se associam à superfície e ao desenho da madeira. Assim, esta propriedade deve ser incorporada ao planejamento, visando à caracterização tecnológica da madeira e de seus produtos reconstituídos (Mori et al., 2005). O sistema CIE (Comission International de L'Eclairage ou Comissão Internacional de Iluminantes) é o mais utilizado para a quantificação e a medição de cor. Este sistema define a sensação da cor baseado em três elementos: a luminosidade, a tonalidade e a saturação (Camargos \& Gonçales, 1999). O valor de luminosidade é representado pelo símbolo $\mathrm{L}^{*} \mathrm{e}$ varia de branco a preto. A tonalidade é representada por dois pigmentos: $\mathrm{a}^{\star}$, que pode variar de verde a vermelho, e $b^{*}$, que pode variar de azul a amarelo (Leão et al., 2005)

Geralmente, aglomerados são colados pela resina ureia formaldeído devido à sua resistência, ao baixo custo e à cor clara (Sellers Jr., 1994). Assim, a cor do produto final está predominantemente ligada apenas à cor da madeira usada. Porém, Iwakiri et al. (2010) sugerem a mistura de diferentes espécies na composição do painel aglomerado com o intuito de ampliar a oferta de matéria-prima, além de consorciar diferentes características físico-químicas das espécies em uma mistura homogênea.

Portanto, este estudo objetivou caracterizar a cor de aglomerados produzidos com Sequoia sempervirens e Pinus taeda em diferentes proporções de mistura, e suas respectivas alterações de cores após exposição à luz natural, durante seis meses. 


\section{MATERIAL E MÉTODOS}

Foram utilizadas, nesta pesquisa, madeiras de Sequoia sempervirens, de árvores de 18 anos coletadas em plantios da Estação Experimental da EPAGRI de São Joaquim-SC, e madeira de Pinus taeda, coletada já na forma de partículas em uma indústria de painéis aglomerados localizada no município de Araucária-PR.

Os painéis aglomerados foram produzidos em escala laboratorial, de acordo com o plano experimental apresentado na Tabela 1.

A resina utilizada foi ureia formaldeído, aplicada em quantidade de $10 \%$ de sólidos em relação ao peso seco das partículas. Resina e parafina (1\% de sólido) foram aplicadas sobre as partículas em uma encoladeira tipo tambor rotatório, por meio de um bico aspersor integrado ao compressor.

O colchão de partículas foi formado em uma caixa vazada com dimensões laterais de $50 \times 50 \mathrm{~cm}$. O cálculo de quantidade de material utilizado para cada chapa foi feito para uma densidade nominal de $0,80 \mathrm{~g} / \mathrm{cm}^{3}$ e dimensões finais de $50 \times 50 \times 1,5 \mathrm{~cm}$. Os painéis foram prensados à temperatura de $160^{\circ} \mathrm{C}$, pressão específica de $40 \mathrm{kgf} / \mathrm{cm}^{3}$ e um tempo de permanência de 8 minutos. Foram produzidos três painéis por tratamento.

Após período de acondicionamento em câmara climática com temperatura aproximada de $20^{\circ} \mathrm{C}$ e umidade relativa de $65 \%$, foram retirados cinco corpos de prova de cada painel, com $5 \mathrm{~cm}$ de largura e 7,5 cm de comprimento, para medição de cor. Para determinação da cor dos aglomerados, utilizou-se o método CIELAB 1976, com o uso do espectrofotômetro de cor modelo CM-5, fabricado pela empresa Konica Minolta com ângulo do observador de $10^{\circ}$.

Tabela 1. Delineamento experimental.

Table 1. Experimental design.

\begin{tabular}{ccc|}
\multirow{2}{*}{ Tratamento } & \multicolumn{2}{c|}{ Proporção de mistura (\%) } \\
\cline { 2 - 3 } & Sequoia sempervirens & Pinus taeda \\
\hline T1 & 100 & 0 \\
T2 & 0 & 100 \\
T3 & 75 & 25 \\
T4 & 50 & 50 \\
T5 & 25 & 75 \\
\hline
\end{tabular}

A medição da cor foi feita em dois diferentes pontos da superfície exposta de cada corpo de prova. No total, foram realizadas 30 medições por tratamento. Os parâmetros colirimétricos obtidos foram: $L^{*}$ (luminosidade), $a^{*}$ (coordenada no eixo vermelho-verde) e $b^{*}$ (coordenada do eixo azul-amarelo). Para o cálculo dos parâmetros C (saturação) e $\mathrm{h}^{\star}$ (ângulo de tinta), foram utilizadas as Equações 1 e 2.

$\mathrm{C}=\left(\mathrm{a}^{\star 2}+\mathrm{b}^{\star 2}\right) / 2$

$\mathrm{h}^{*}=\tan ^{-1}\left(\mathrm{~b}^{\star} / \mathrm{a}^{*}\right)$

Para mensurar as alterações na cor, foi utilizada a Equação 3

$\Delta \mathrm{E}=\sqrt{\Delta L^{2}+\Delta a^{2}+\Delta b^{2}}$

Os corpos de prova foram expostos a um ângulo de $45^{\circ}$ e orientados no eixo leste-oeste, voltado para o norte, seguindo uma adaptação da norma europeia EN 927-3 ao intemperismo natural, indicado na Figura 1. O teste teve início no dia 11 de abril de 2012 e foi montado no município de Curitiba-PR (latitude: -25,449039; longitude: -49,238221). As demais medições ocorreram no dia 15 de maio, 13 de agosto e 9 de outubro de 2012.

Para avaliar o efeito do intemperismo sobre os parâmetros colorimétricos dos aglomerados, fez-se uma análise de variância e, quando significativa, foi aplicado um teste de diferença de médias de Tukey a $5 \%$ de probabilidade.

\section{RESULTADOS E DISCUSSÃO}

A Tabela 2 apresenta os valores médios dos parâmetros colorimétricos das superfícies dos diferentes tratamentos de aglomerados, produzidos com diferentes quantidades de madeira de Pinus taeda e Sequoia sempervirens.

Observa-se que todos os parâmetros colorimétricos apresentaram efeito significativo entre aglomerados produzidos com diferentes proporções de partículas de Sequoia sempervirens. Todos os aglomerados apresentaram valores de $\mathrm{a}^{*} \mathrm{e}$ $\mathrm{b}^{\star}$ positivos, seguindo o mesmo padrão colorimétrico da madeira sólida descrito por Camargos \& Gonçalez (1999). 
Os aglomerados feitos com $100 \%$ de madeira de Sequoia sempervirens apresentam uma cor vermelha amarronzada, que é resultado dos valores mais altos do pigmento vermelho $\left(\mathrm{a}^{*}\right)$ e dos valores do pigmento amarelo $\left(b^{*}\right)$, que, embora sejam os mais baixos em comparação aos demais aglomerados, ainda são superiores ao valor médio de $a^{*}$. Para os aglomerados feitos exclusivamente de partículas de Pinus taeda, o parâmetro determinante foi $\mathrm{o} \mathrm{b}^{\star}$, em detrimento do baixo valor de $\mathrm{a}^{\star}$, que leva o painel a ser classificado como de cor amarelada. $\mathrm{O}$ ângulo de tinta $\left(\mathrm{h}^{*}\right)$ também ajuda a definir o padrão de cor do aglomerado de $P$. taeda, já que seu alto valor faz o aglomerado se aproximar do eixo $b^{*}$. A saturação da cor $(\mathrm{C})$ também é mais evidente em aglomerados de partículas de $S$. sempervirens do que daqueles de partículas de $P$. taeda. Os valores médios de todos os parâmetros dos aglomerados produzidos com partículas de Pinus taeda mostram-se semelhantes aos apresentados por Castro et al. (2013) para partículas da mesma espécie. Essa semelhança indica que o aglomerado mantém as características colorimétricas da madeira utilizada em sua formação, com pouca influência do adesivo ureia-formaldeído.

Os tratamentos feitos a partir de misturas de espécies apresentaram parâmetros intermediários entre os aglomerados produzidos com partículas de apenas uma espécie. $\mathrm{O}$ aumento da proporção de partículas de Pinus taeda tende a diminuir a influência do eixo vermelho $\left(a^{*}\right)$ e aumentar a do eixo amarelo $\left(b^{\star}\right)$, fato confirmado com o aumento do ângulo de tinta $\left(\mathrm{h}^{\star}\right)$.

O valor da luminosidade $\left(\mathrm{L}^{*}\right)$ foi crescente, de acordo com o aumento da proporção de partículas de Pinus taeda na mistura. Isso indica que o acréscimo de partículas de Sequoia sempervirens na mistura tende a produzir aglomerados mais escuros. Porém, uma vez que todos os valores de $\mathrm{L}^{*}$ apresentaram valores acima de 56, independentemente de sua composição, os aglomerados podem ser classificados como cores claras (Camargos \& Gonçalez, 1999).
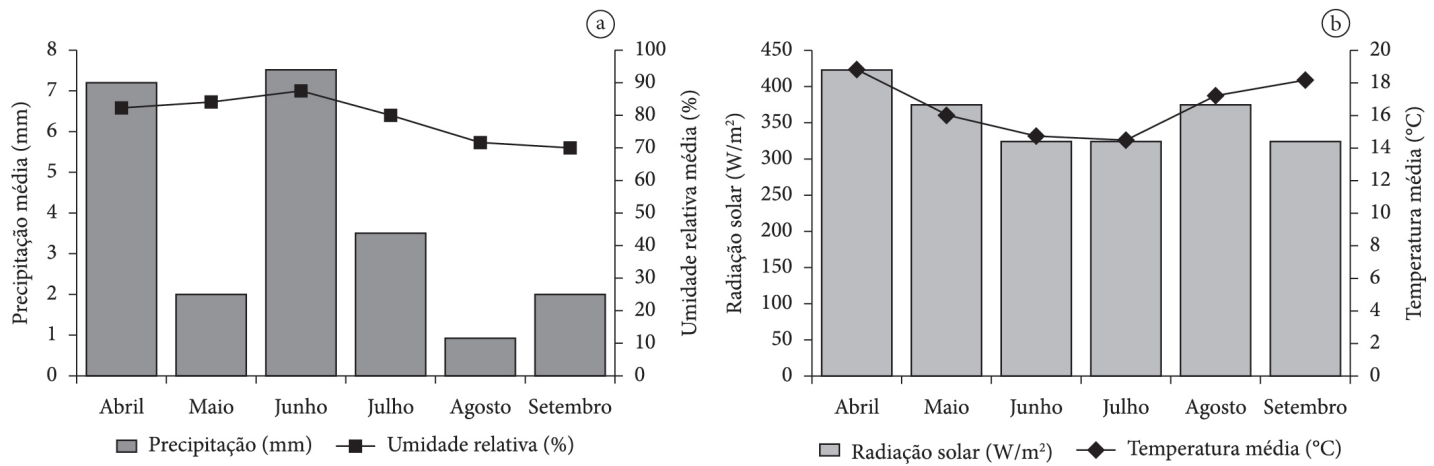

Figura 1. Variação das condições meteorológicas de precipitação, umidade relativa, radiação solar e temperatura, durante o período do teste.

Figure 1. Meteorological conditions during the experiment.

Tabela 2. Valores médios dos parâmetros colorimétricos das amostras dos aglomerados das madeiras de Pinus taeda e Sequoia sempervirens.

Table 2. Average values of colorimetric parameters from Pinus taeda and Sequoia sempervirens particleboards.

\begin{tabular}{cccccc}
\multirow{2}{*}{$\%$ de S. sempervirens } & \multicolumn{5}{c}{ Parâmetros colorimétricos } \\
\cline { 2 - 6 } & $\mathbf{L}^{\star}$ & $\mathbf{a}^{\star}$ & $\mathbf{b}^{\star}$ & $\mathbf{C}$ & $\mathbf{h}^{\star}$ \\
\hline 100 & $59,81^{\mathrm{e}}(1,34)$ & $11,89^{\mathrm{a}}(4,49)$ & $22,83^{\mathrm{e}}(1,50)$ & $25,75^{\mathrm{e}}(1,50)$ & $62,51^{\mathrm{e}}(1,78)$ \\
0 & $68,50^{\mathrm{a}}(1,09)$ & $7,18^{\mathrm{e}}(3,44)$ & $27,76^{\mathrm{a}}(2,34)$ & $28,67^{\mathrm{a}}(2,29)$ & $75,49^{\mathrm{a}}(0,61)$ \\
75 & $61,44^{\mathrm{d}}(1,30)$ & $10,77^{\mathrm{b}}(3,30)$ & $24,09^{\mathrm{d}}(1,93)$ & $26,39^{\mathrm{d}}(1,58)$ & $65,91^{\mathrm{d}}(1,34)$ \\
\hline 50 & $63,84^{\mathrm{c}}(1,08)$ & $9,33^{\mathrm{c}}(3,89)$ & $25,45^{\mathrm{c}}(1,81)$ & $27,11^{\mathrm{c}}(1,49)$ & $69,85^{\mathrm{c}}(1,29)$ \\
\hline 25 & $66,39^{\mathrm{b}}(4,76)$ & $8,46^{\mathrm{d}}(3,51)$ & $26,36^{\mathrm{b}}(1,43)$ & $27,69^{\mathrm{b}}(1,31)$ & $72,20^{\mathrm{b}}(0,90)$ \\
\hline
\end{tabular}

${ }^{*}$ Médias seguidas pela mesma letra, dentro da mesma coluna, não se diferenciam estatisticamente no nível de $5 \%$ de probabilidade pelo teste de Tukey; ${ }^{*}$ Os valores dentro dos parênteses são relativos aos coeficientes de variância (\%). 
A Tabela 3 apresenta a variação da cor e de seus parâmetros quando misturaram-se diferentes proporções de partículas de Pinus taeda se comparado com o aglomerado feito exclusivamente de partículas de Sequoia sempervirens.

Observa-se que, à medida que aumenta a proporção das partículas da madeira de Pinus taeda na mistura, a tendência é que resultado final seja mais claro $\left(\Delta \mathrm{L}^{*}\right)$, além de o parâmetro que indica o pigmento vermelho $\left(\Delta \mathrm{a}^{*}\right)$ diminuir na mesma dimensão que o pigmento amarelo $\left(\Delta \mathrm{b}^{\star}\right)$ aumentou. Ressalta-se que a variação total da cor $(\Delta \mathrm{E})$ pode ser comprovada pela alteração gradativa da cor, de acordo com a diminuição do uso das partículas da madeira de Sequoia sempervirens.

A Figura 2 apresenta os valores médios de $L^{*}$ para todos os aglomerados durante o processo de envelhecimento natural. Colunas da mesma cor acompanhadas de mesmas letras indicam que não houve diferença estatística entre médias.

Para os aglomerados produzidos com apenas uma espécie, seja Pinus taeda ou Sequoia sempervirens, e para aglomerados produzidos com $50 \%$ de cada espécie, a luminosidade se estabilizou após quatro meses de envelhecimento natural. Porém, para os aglomerados com misturas desiguais, os valores médios de $\mathrm{L}^{*}$ continuaram a diminuir após o quarto mês. Outro comportamento que pode ser observado é que os valores de luminosidade inicialmente distintos para aglomerados com diferentes misturas tendem a apresentar o mesmo grau de escurecimento após seis meses.

O padrão do escurecimento com uma queda inicial dos valores médios do parâmetro $L^{*}$ seguida de uma tendência de estabilização também foi observado por outros autores, para madeiras sólidas. Por exemplo: Lima et al. (2013) observaram um valor médio inicial de $\mathrm{L}^{*}$ de 70,32 para lâminas de madeira de Balfourondendron riedelianum, naturalmente de cor amarelo-claro, semelhante ao Pinus taeda, após 120 horas de exposição à radiação UV; este valor foi reduzido para 63,56, mantendo-se relativamente constante até 500 horas de radiação.

A Figura 3 apresenta a variação da tonalidade vermelha $\left(\mathrm{a}^{\star}\right)$ para os tratamentos após seis meses de envelhecimento natural. Colunas da mesma cor,

Tabela 3. Variações ocorridas entre parâmetros colorimétricos de aglomerados de mistura de partículas em relação ao aglomerado de Sequoia sempervirens

Table 3. Changes of the colorimetric parameters of particles manufactures with blend of particles compared with Sequoia sempervirens particleboard.

\begin{tabular}{|c|c|c|c|c|}
\hline & $\Delta \mathbf{L}^{*}$ & $\Delta \mathbf{a}^{\star}$ & $\Delta \mathbf{b}^{*}$ & $\Delta \mathrm{E}$ \\
\hline T3 & 1,636 & $-1,119$ & 1,257 & 2,347 \\
\hline $\mathrm{T} 4$ & 4,035 & $-2,554$ & 2,615 & 5,444 \\
\hline T5 & 6,588 & $-3,425$ & 3,530 & 8,221 \\
\hline
\end{tabular}

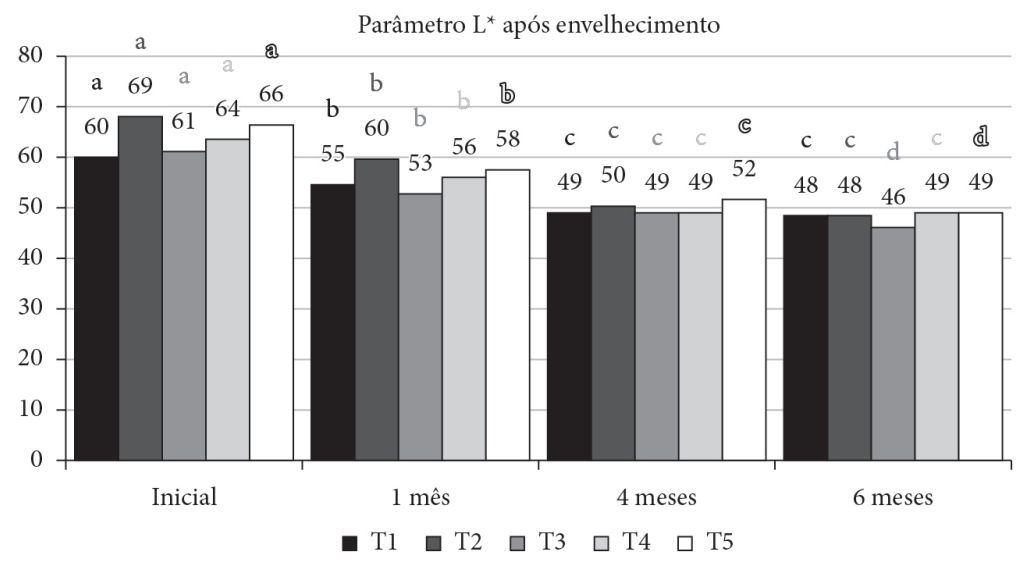

Figura 2. Parâmetro colorimétrico $L^{*}$ após 1,4 e 6 meses de envelhecimento natural.

Figure $2 . \mathrm{L}^{\star}$ parameter change after 1,4 and 6 months of outdoor weathering. 
acompanhadas de letras iguais, indicam que não houve diferença estatística entre médias.

Para todos os tratamentos, houve uma redução na intensidade da tonalidade vermelha. Os aglomerados produzidos com partículas de Sequoia sempervirens, inicialmente, apresentaram maiores valores para o parâmetro $\mathrm{a}^{\star}$, indicando tons mais avermelhados, em comparação com os apresentados pelos aglomerados de Pinus taeda. Porém, com o decorrer do envelhecimento, houve uma tendência de homogeneização dos valores de $\mathrm{a}^{*}$ para todos os aglomerados.

Martins et al. (2011) estudaram o efeito do envelhecimento artificial na cor de madeira sólida de Eucalyptus benthamii, que naturalmente possui uma tonalidade avermelhada, como a Sequoia sempervirens, e o Pinus caribaea, de cor semelhante ao Pinus taeda. A madeira sólida de E. benthamii apresentou um aumento pronunciado no valor médio de $a^{*}$ nas primeiras 42 horas de fotodegradação, se estabilizando nas horas seguintes. Já a madeira de P. caribaea apresentou um aumento moderado e constante desse parâmetro durante as 168 horas de exposição. O comportamento inverso, observado nos aglomerados expostos ao ar livre, indica que a alteração química causada pela exposição exclusiva de raios ultravioleta não é forte o suficiente para impedir a degradação da pigmentação das cores por outras variáveis presentes no intemperismo natural.

O grau de intensidade da tonalidade amarela $\left(b^{*}\right)$ nos aglomerados durante o processo de envelhecimento é mostrado na Figura 4.

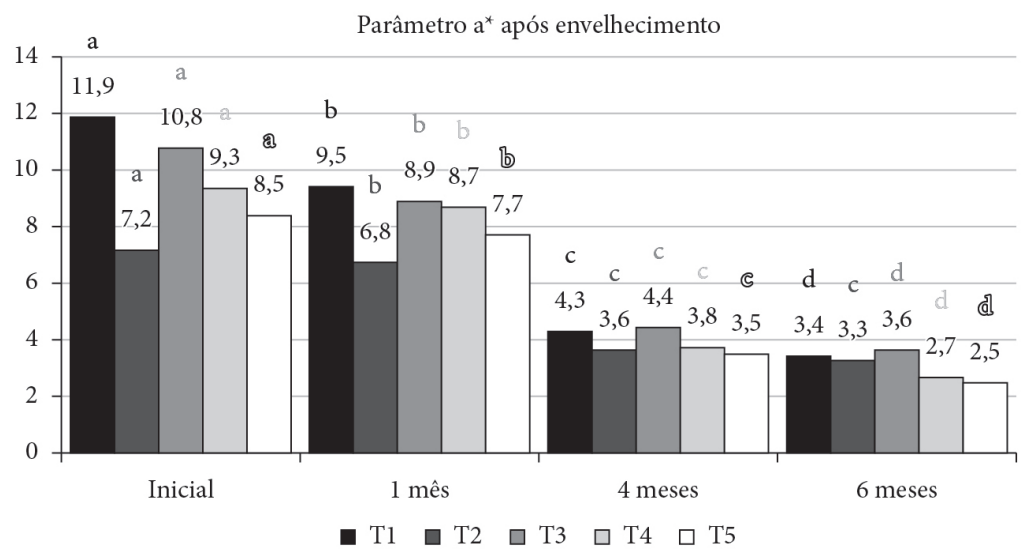

Figura 3. Parâmetro colorimétrico a após 1,4 e 6 meses de envelhecimento natural.

Figure 3. $a^{\star}$ parameter change after 1, 4 and 6 months of outdoor weathering.

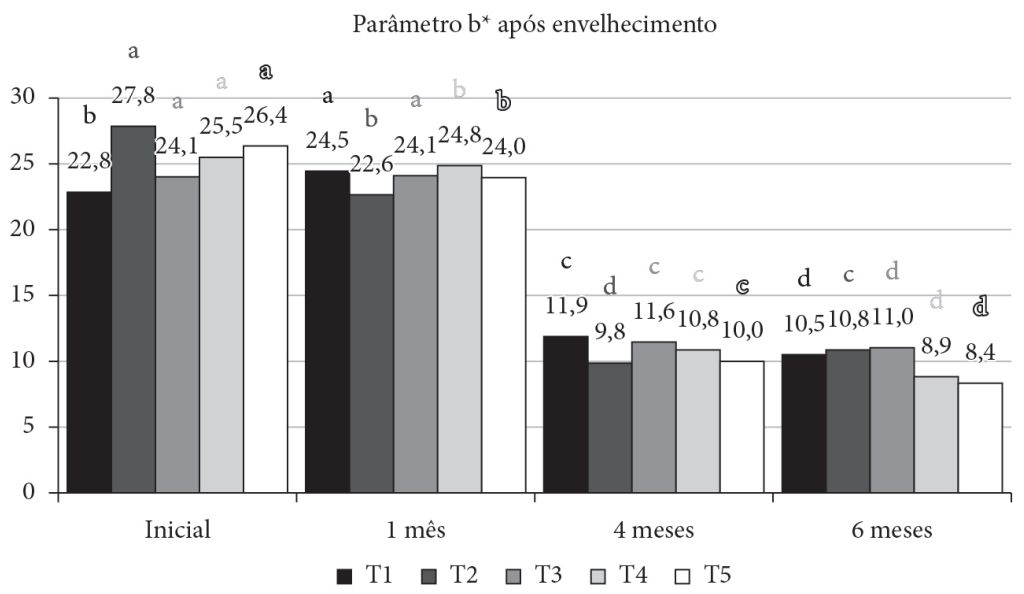

Figura 4. Parâmetro colorimétrico $b^{\star}$ após 1,4 e 6 meses de envelhecimento natural. Figure $4 . b^{\star}$ parameter change after 1,4 and 6 months of outdoor weathering. 
$\mathrm{O}$ parâmetro $\mathrm{b}^{\star}$ apresentou um comportamento semelhante ao da tonalidade vermelha $\left(a^{*}\right)$, com uma redução drástica dos valores médios nos primeiros 4 meses, seguida de uma tendência de estabilização. Assim como o parâmetro a*, Martins et al. (2011) observaram um padrão inverso em madeira sólida após radiação de ultravioleta, com um ganho no parâmetro $b^{\star}$. Inicialmente, todos os tratamentos apresentavam diferentes níveis de pigmentação amarela; entretanto, após 6 meses de envelhecimento natural, os aglomerados feitos exclusivamente de Sequoia sempervirens ou de Pinus taeda, assim como aqueles feitos com mistura de 50\% de cada um, apresentaram valores estatisticamente iguais.

A Figura 5 apresenta a variação do parâmetro C, relativo à saturação da cor dos aglomerados durante os seis meses de envelhecimento natural. Colunas com a mesma cor e acompanhadas de letras semelhantes indicam que não houve diferença estatística entre os valores médios de C durante o processo.

Os valores médios de saturação dos aglomerados sofreram uma queda significativa após o primeiro mês de exposição às intempéries. Os aglomerados feitos de partículas de Sequoia sempervirens não sofreram degradação deste parâmetro no primeiro mês, mas acompanharam a queda entre os meses 1 e 4, que os outros aglomerados sofreram. Embora apenas os aglomerados produzidos exclusivamente com partículas de Pinus taeda tenham se estabilizado em relação ao parâmetro após quatro meses, houve uma tendência de estabilização de todos os tratamentos neste período. A mesma tendência também foi observada por Ghosh et al. (2009), quando estudaram o envelhecimento natural de painéis de Pinus sylvestris. Os autores observaram que o parâmetro $\mathrm{C}$ sofreu uma redução máxima nos primeiros três meses e tendeu a se estabilizar em medições feitas até um ano de exposição.

A Figura 6 apresenta os valores de alteração total de cor $(\Delta \mathrm{E})$, comparando aglomerados após 1, 4 e 6 meses de exposição à luz natural com suas contrapartes não envelhecidas.

A influência do intemperismo ao longo de seis meses sobre os diferentes tratamentos com amostras de aglomerados de Pinus taeda e Sequoia sempervirens foi clara. A alteração total de cor foi mais drástica nos primeiros quatro meses e tendeu a uma estabilização nos períodos seguintes. Os valores médios de $\Delta \mathrm{E}$ foram maiores para os aglomerados com maior quantidade de partículas de Pinus taeda, indicando uma sensibilidade maior dos aglomerados de tons mais amarelados às mudanças na cor devidas à exposição à luz natural e às variações climáticas. Como pode ser visto na Figura 7, a aparência visual dos aglomerados pode ser controlada pela proporção da mistura de partículas no processo de produção. Porém, após um período de envelhecimento natural, a tendência de todos os aglomerados é ter a mesma coloração.

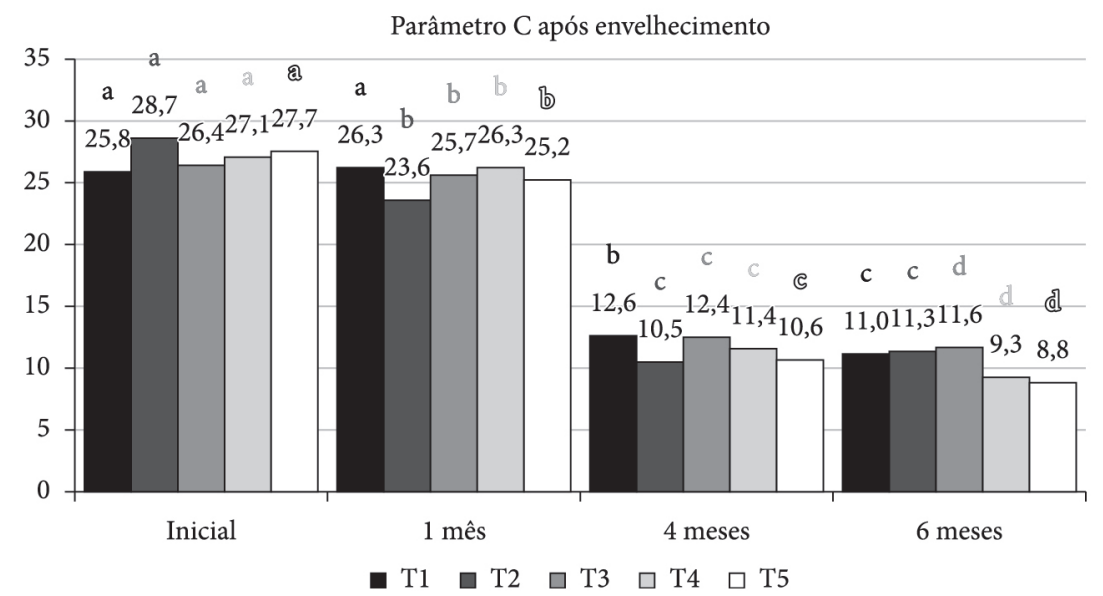

Figura 5. Parâmetro colorimétrico C após 1, 4 e 6 meses de envelhecimento natural. Figure 5. C parameter change after 1, 4 and 6 months of outdoor weathering. 


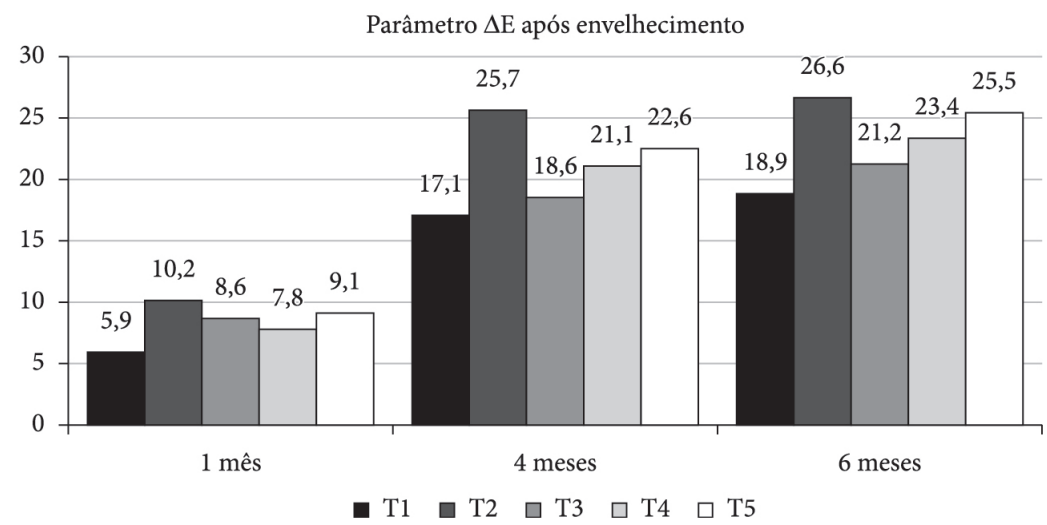

Figura 6. Parâmetro colorimétrico $\Delta \mathrm{E}$ após 1,4 e 6 meses de envelhecimento natural.

Figure 6. $\Delta \mathrm{E}$ parameter change after 1,4 and 6 months of outdoor weathering.

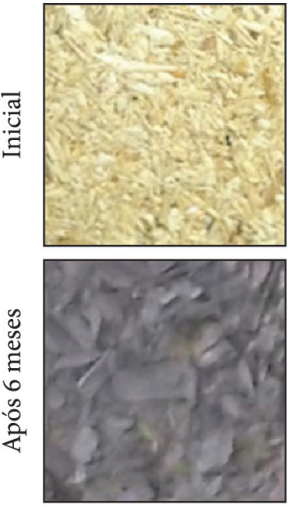

$\mathrm{T} 1$
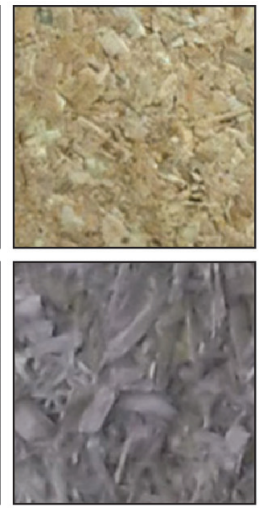

$\mathrm{T} 2$
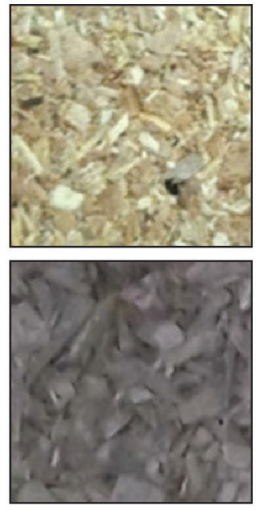

T3

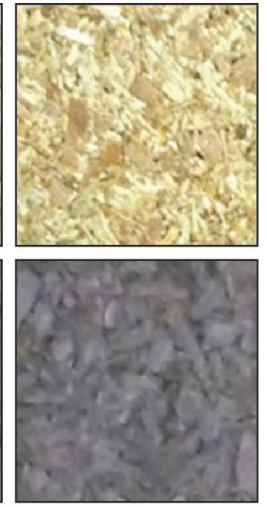

T4

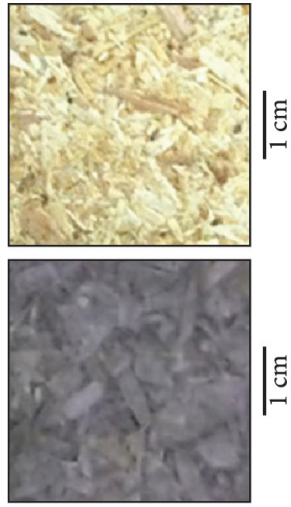

T5

Figura 7. Aparência visual inicial dos tratamentos e após 6 meses de envelhecimento natural.

Figure 7. Impression of the treatments after outdoor weathering over six months.

\section{CONCLUSÃO}

Com base nos resultados obtidos nesta pesquisa, as seguintes conclusões podem ser apresentadas:

- Aglomerados tendem a manter a aparência colorimétrica da espécie das partículas utilizadas no processo. Aglomerados de Sequoia sempervirens apresentam uma coloração avermelhada, enquanto que os feitos com partículas de Pinus taeda apresentam uma cor amarelada. Dessa forma, a indústria de aglomerados pode apresentar produtos com novos padrões de cores a partir de uma produção com mistura de partículas de diferentes espécies;

- A exposição ao envelhecimento natural acarreta uma diminuição brusca das pigmentações, tanto vermelha quanto amarela, após os primeiros quatro meses. Depois deste período inicial, os parâmetros colorimétricos tendem a se estabilizar em uma cor única acinzentada para todas as cinco tonalidades de aglomerados estudadas.

\section{STATUS DA SUBMISSÃO}

Recebido: 19 nov., 2012

Aceito: 28 jul., 2014

Publicado: 21 nov., 2014

\section{AUTOR(ES) PARA CORRESPONDÊNCIA}

\section{Vinicius Gomes de Castro}

Departamento de Engenharia e Tecnologia

Florestal, Universidade Federal do Paraná UFPR, CEP 80210-170, Curitiba, PR, Brasil e-mail: vinnie.castro@gmail.com 


\section{REFERENNCIAS}

Camargos JAA, Gonçalez JC. A colorimetria aplicada como instrumento na elaboração de uma tabela de cores de madeira. Brasil Florestal 1999; 71: 30-41.

Castro VG, Klock U, Iwakiri S, Bolzon GI. Avaliação colorimétrica de partículas de Pinus taeda submetidas a diferentes métodos de acetilação. Scientia Forestalis 2013; 41(98): 265-270.

European Committee for Standardization - CEN. EN 927-3: paints and varnishes: coating materials and coating systems for exterior wood. Part 3: natural weathering test. Brussels; 2000.

Ghosh SC, Militz H, Mai C. Natural weathering os Scott Pine (Pinus sylvestris L.) boards modified with functionalised commercial silicone emulsions. BioResources 2009; 4(2): 659-673.

Iwakiri S, Andrade AS, Cardoso AA Jr, Chipanski ER, Prata JG, Adriazola MKO. Produção de painéis aglomerados de alta densificação com uso de resina melamina-uréiaformaldeído. Cerne 2005; 11(4): 323-328.

Iwakiri S, Zeller F, Pinto JÁ, Ramirez MGL, Souza MM, Seixas R. Avaliação do potencial de utilização da madeira de Schizolobium amazonicum "Paricá" e Cecropia hololeuca "Embaúba" para produção de painéis aglomerados. Acta Amazonica 2010; 40(2): 303-308. http://dx.doi.org/10.1590/S0044-59672010000200008

Leão AC, Araújo AA, Souza LAC. Implementação de sistema de gerenciamento de cores para imagens digitais. In: Teixeira A, Barrére E, Abrão IC. Web e multimídia: desafios e soluções. Poços de Caldas: PUCMinas; 2005.

Lima CL, Gonçalez JC, Costa TRV, Pereira RS, Lima JBM, Lima MAS. Comportamento da cor de lâminas de madeira de Pau-marfim (Balfourodendron riedelianum) tratada com produtos de acabamento. Revista Árvore
2013; 37(2): 377-384. http://dx.doi.org/10.1590/S010067622013000200020

Martins SA, Santos CMT, Gonçalez JC, Camargos JAA. Envelhecimento artificial acelerado por radiação ultravioleta de madeiras de Eucalyptus benthamii e Pinus caribaea var. hondurensis. Floresta 2011; 41(1): 87-96.

Mattos RLG, Gonçalves RM, Chagas FB. Painéis de madeira no Brasil: panorama e perspectivas. BNDS Setorial 2008; (27): 121-156.

Mori CLSO, Lima JT, Mori FA, Trugilho PF, Gonçalvez JC. Caracterização da cor da madeira de clones de híbridos de Eucaluptus spp. Cerne 2005; 11(2):137-146.

Nascimento MF. $C P H$ : chapas de partículas homogêneas: madeiras do nordeste do Brasil [tese]. São Carlos: Universidade de São Paulo; 2003.

Rosa V, Gonçalves B. Cor sustentável: solução que não agridem o meio ambiente no design de móveis. In: Anais do IX Congresso Brasileiro de Pesquisa e Desenvolvimento em Design; 2010; São Paulo. São Paulo: Universidade Anhembi Morumbi; 2010. p. 5324-5330.

Sellers T Jr. Adhesives in the wood industry. In: Pizzi A, Mittal KL. Handbook of adhesive technology. New York: Marcel Decker; 1994.

Solyon, GJP. Minimização e reaproveitamento de resíduos de uma indústria de tintas e impressão de papéis decorativos [dissertação]. Curitiba: Universidade Federal do Paraná; 2009.

Tsunetsugu Y, Miyazaki Y, Sato H. Physiological effects in human induced by the visual stimulation of room interiors with different wood quantities. Japan Wood Research Societ 2007; 53(1): 11-16.

Zaleski, C. B. Materiais e conforto: um estudo sobre a preferência por alguns materiais de acabamento e sua relação com o conforto percebido em interiores residenciais da classe média de Curitiba [dissertação]. Curitiba: Universidade Federal do Paraná; 2006. 